\title{
Nachhaltigkeitsdefizite integriert angehen
}

\section{Befindet sich Deutschland auf dem Weg zur Nachhaltigkeit? Eine Studie der Helmholtz-Gemeinschaft sagt ganz klar nein. Bisher eingeleitete Maßnahmen reichten bei weitem nicht aus, ökologische, ökonomische und soziale Nachhal- tigkeitsziele in absehbarer Zeit zu erreichen. Es werden deshalb tief greifende weitere Schritte und ein integratives und simultanes Vorgehen bei der lösung verschiedener Nachhaltigkeitsprobleme gefordert.}

$\mathrm{V}$

Von Reinhard Coenen, Juliane Jörissen und Jürgen Kopfmüller erschiedene Forschungsgruppen der Helmholtz-Gemeinschaft haben unter Federführung des Instituts für Technikfolgenabschätzung und Systemanalyse den Bericht „Nachhaltigkeitsprobleme in Deutschland" vorgelegt, der die wesentlichen Ergebnisse des Verbundprojekts ,Global zukunftsfähige Entwicklung - Perspektiven für Deutschland“ zusammenführt (1). Das Projekt spannt den Bogen von der Operationalisierung des Leitbilds über die Diagnose der größten Nachhaltigkeitsdefizite Deutschlands, die Vorausschau in Szenarien bis zu Handlungsoptionen zur Erreichung von Nachhaltigkeitszielen.

Theoretische Basis des Vorhabens ist das integrative Konzept nachhaltiger Entwicklung, das ökologische, ökonomische, soziale und politisch-institutionelle Aspekte unter Berücksichtigung der globalen Perspektive zusammenführt. Ausgehend von drei konstitutiven Elementen des Leitbilds, inter- und intragenerative Gerechtigkeit, globale Orientierung, anthropozentrischer Ansatz, werden drei generelle Ziele nachhaltiger Entwicklung herausgearbeitet: Sicherung der menschlichen Existenz, Erhaltung des gesellschaftlichen Produktivpotenzials und Bewahrung gesellschaftlicher Entwicklungs- und Handlungsmöglichkeiten. Diese Ziele werden durch 15 substantielle Nachhaltigkeitsregeln, die Mindestanforderungen zum Beispiel in Bezug auf die Ressourcennutzung, die Sach-, Human- und Wissenskapitalausstattung oder den Erhalt sozialer und kultureller Ressourcen benennen, näher bestimmt und durch zehn instrumentelle Regeln ergänzt, die wesentliche Rahmenbedingungen für Nachhaltigkeit definieren (2). Kon- kretisiert durch Indikatoren bietet dieses Regelsystem ein Rahmenkonzept zur Bewertung des Grades nachhaltiger Entwicklung.

\section{Die größten}

\section{Nachhaltigkeitsdefizite}

Für diese Indikatoren werden bereits im politischen Raum formulierte oder im Projekt definierte Zielwerte benannt. Auf Basis von Soll-IstVergleichen werden als gegenwärtig drängendste Nachhaltigkeitsprobleme Deutschlands benannt: Armut, drastische globale Einkommensunterschiede, Arbeitslosigkeit, Bildungsdefizite, mangelnde Chancengleichheit, Flächenverbrauch, Biodiversitätsrückgang, Belastung der Waldböden, Abbau nicht-erneuerbarer Ressourcen, Klimawandel, ungleiche globale Verteilung der Umweltnutzungsmöglichkeiten, Gewässerverschmutzung, umweltbedingte Gesundheitsbeeinträchtigungen (Feinstaubemissionen, Ozon, Lärm) Staatsverschuldung und mangelnde Wahrnehmung globaler Verantwortung. Für die Aktivitätsbeziehungsweise Bedürfnisfelder Mobilität und Verkehr, Wohnen und Bauen, Ernährung und Landwirtschaft sowie Freizeit und Tourismus werden entsprechende Analysen durchgeführt.

Explorative Szenarien sind ein bewährtes Instrument, mit Unsicherheiten über die künftige politisch-gesellschaftliche Entwicklung, die künftige Nachhaltigkeitssituation sowie Handlungsbedarfe umzugehen. Sie beschreiben denkbare gesellschaftliche Entwicklungspfade auf der Basis unterschiedlicher Annahmen über als wesentlich erachtete politisch-gesellschaftliche Strömungen, auch Deskriptoren genannt. Im Projekt werden folgende Deskriptoren verwendet: der Grad der globalen wirtschaftlichen Verflechtung und die Rolle des Markts als Steuerungsmechanismus, die
Bedeutung (sub)nationaler und supranationaler Politikebenen, die Entwicklung des Sozialstaats, die Entwicklung von Werthaltungen sowie der Grad der Teilhabe an gesellschaftlichen Entscheidungsprozessen. Es werden drei Szenarien für Deutschland erarbeitet, wobei gleichgerichtete Entwicklungen in anderen Industrieländern unterstellt werden.

\section{Drei Szenarien}

Beim Szenario Dominanter Markt (DOM) wird von einer sich weiter verstärkenden wirtschaftlichen Globalisierung und einer dominanten Rolle des Marktes als Steuerungsmechanismus ausgegangen. Als Folge des globalisierungsbedingt scharfen Standortwettbewerbs wird angenommen, dass nationale Regierungen versuchen, die Standortbedingungen zu verbessern: zum Beispiel durch einseitige Entlastung der Unternehmen bei Steuern und Sozialbeiträgen, reduzierte Sozialleistungen oder Deregulierung vor allem auf dem Arbeitsmarkt. Die Umweltpolitik ist durch no/ low-regret-Maßnahmen geprägt.

Im Szenario Modernisierung in Zeiten der Globalisierung (MOD) wird von einer proaktiven Politik ausgegangen, die Chancen der Globalisierung zu nutzen und unerwünschte Folgen zu mildern versucht. Kennzeichen ist eine Gratwanderung zwischen der Stärkung internationaler Wettbewerbsfähigkeit und der Gewährleistung sozialer Gerechtigkeit. Die Folge sind inkrementelle Veränderungen der sozialen Sicherungssysteme, Arbeitgeber- und Arbeitnehmerbeiträge werden gleichmäßig moderat abgesenkt, bei staatlicher Förderung privater Vorsorge. Umweltpolitik erfolgt in langsamem internationalem Gleichschritt, da eine Vorreiterrolle als Gefährdung der Wettbewerbsfähigkeit gesehen wird.

Das Szenario Regionalisierung und Gemeinwohlorientierung (REGIO) unterstellt deutliche Veränderungen von Wertvorstellungen in Richtung Gemeinwohlorientierung und Umweltschutz. $\mathrm{Zu}-$ gleich erfolgt eine Rückbesinnung auf nationale und lokale Wirtschaftsstrukturen. Es wächst die Bereitschaft zu grundlegenden Reformen. Angenommen werden die Abkopplung der sozialen Sicherung von der abhängigen Erwerbstätigkeit und verstärkte staatliche Förderung gesellschaftlicher Arbeit. Einschneidende umweltpolitische Maßnahmen, insbesondere im Bereich Klimaschutz und Flächenverbrauch, werden akzeptiert.

Um die nachhaltigkeitsrelevanten Auswirkungen dieser Szenarien abzuschätzen, werden, soweit 
möglich, Simulationsrechnungen mit dem umweltökonomischen Modell PANTA RHEI der Gesellschaft für Wirtschaftliche Strukturforschung bis 2020 durchgeführt. Danach würden sich im Szenario DOM ökologische Nachhaltigkeitsdefizite noch verschärfen. Im ökonomisch-sozialen Bereich würden sich voraussichtlich Unterschiede in der Einkommens- und Vermögensverteilung und damit Armutsprobleme verstärken. Die Arbeitslosigkeit würde sich kaum verringern. Beim Szenario MOD nähert man sich Nachhaltigkeitszielen im sozialen und ökologischen Bereich an - allerdings nicht in ausreichendem Maße. Zwar wird das Kyoto-Ziel bei den Kohlendioxid-Emissionen erreicht, anspruchsvollere Ziele für 2020, eine Reduktion um 40 Prozent, aber nicht. Der Flächenverbrauch verharrt auf viel zu hohem Niveau gemessen am Ziel der deutschen Nachhaltigkeitsstrategie von 30 Hektar pro Tag. Die Verbesserungen bei der Arbeitslosigkeit würden begrenzt sein. Beim Szenario REGIO werden zwar die ökologischen und sozialen Nachhaltigkeitsziele erreicht; die sich zeigende Wachstumsschwäche und kritische Entwicklungen bei anderen ökonomischen Indikatoren lassen jedoch wirtschaftliche Verwerfungen nach 2020 erwarten, so dass in Frage zu stellen ist, ob es sich um einen nachhaltigen Entwicklungspfad handeln könnte.

\section{- Wie ist mehr Nachhaltigkeit mög- lich?}

Die Ergebnisse und einzelne „Optimierungsrechnungen“ in den Szenarien zeigen, dass es Maßnahmen erheblicher Eingriffstiefe bedarf, um Deutschland auf einen Nachhaltigkeitspfad zu lenken. Welche Maßnahmen in Zukunft wie intensiv eingesetzt werden können, hängt von den dann herrschenden politisch-gesellschaftlichen Strömungen ab. Deshalb werden für zentrale Nachhaltigkeitsdefizite jeweils zwei alternative Maßnahmenbündel erarbeitet. Die eine Gruppe von Bündeln umfasst Maßnahmen, die Aussicht auf Durchsetzbarkeit hätten, wenn die künftige Entwicklung in Richtung der Szenarien Dom und MOD ginge. Die andere Gruppe bezieht sich entsprechend auf die Szenarien MOD und REGIO. Diese beiden Handlungsoptionen repräsentieren verschiedene Grundausrichtungen einer Nachhaltigkeitspolitik.

Die Maßnahmenbündel „DOM/MOD“ sind gekennzeichnet durch Dominanz des Marktes als Steuerungsmechanismus, möglichst wenig staatliche Eingriffe sowie Deregulierungen. Die Maßnahmenbündel „MOD/REGIO“ enthalten dagegen andere und stärkere Lenkungsmechanismen des Staates, vor allem in Form von Steuern/Abgaben auf nicht-nachhaltige Aktivitäten, mit denen nachhaltigkeitsfördernde Maßnahmen finanziert werden können, sowie finanzielle Kompensationen für die negativ Betroffenen. So stehen zum Beispiel bei den Maßnahmenbündeln DOM/MOD für die Probleme Armut und Langzeitarbeitslosigkeit Maßnahmen im Vordergrund, die auf stärkere Eigenverantwortung und Aktivierung setzen, die durch den Abbau sozialstaatlicher Leistungen befördert werden soll. Gleichzeitig werden dadurch Unternehmen kostenmäßig entlastet, sowie Maßnahmen zur Deregulierung vor allem des Arbeitsmarktes eingeleitet. Dagegen enthalten die Maßnahmenbündel MOD/REGIO strukturelle Veränderungen der sozialen Sicherungssysteme und des Arbeitsmarkts wie Bürgerrente, direkte Schaffung von Arbeitsplätzen im gemeinwirtschaftlichen Bereich etc.

Im ökologischen Bereich bauen beide Maßnahmenbündel auf einer breiten Palette bereits implementierter Maßnahmen auf, insbesondere ordnungsrechtlicher, informatorischer und kooperativer Art. Die Szenariosimulationen legen aber nahe, dass deren Effekte nicht ausreichen, um wichtige Nachhaltigkeitsziele zu erreichen. Deshalb werden in beiden Bündeln ökonomische Instrumente ergänzt. Beim Bündel DOM/MOD stehen mengensteuernde Instrumente im Vordergrund, wie Zertifikatshandel in verschiedenen Bereichen. Der Staat gibt hier nur Reduktionsziele und die Rahmenbedingungen des Handels vor. Im Bündel MOD/REGIO stehen dagegen preissteuernde ökonomische Instrumente wie Steuern und Abgaben im Vordergrund: etwa die Fortschreibung und der Ausbau der Ökosteuer im Sinne einer Kohlendioxid-Steuer, die Weiterführung des Erneuerbare-Energien-Gesetzes sowie hohe Straßennutzungsgebühren für Lkw; eine kombinierte Bodenwert- und Bodenflächensteuer als Ersatz für die Grundsteuer sowie eine Versiegelungsabgabe. Die Einnahmen könnten für die Lösung anderer Nachhaltigkeitsdefizite eingesetzt werden. Im Ergebnis ist bei den Bündeln zu DOM/MOD wegen deren geringer Eingriffstiefe mit der Erreichung politisch formulierter Nachhaltigkeitsziele bestenfalls nach 2020 zu rechnen. Bei den Bündeln zu MOD/REGIO erscheint eine Erreichung wichtiger Nachhaltigkeitsziele bis 2020 eher möglich, allerdings sind hier bezüglich der Akzeptanz der unterstellten hohen Eingriffstiefe Fragezeichen angebracht.

\section{- Proaktiver Staat ist nötig}

Eine wesentliche Erkenntnis des Projekts ist, dass eine erfolgversprechende Nachhaltigkeitsstrategie die Lösung der wesentlichen Probleme simultan und integriert angehen muss. Hierzu kann sich der Staat nicht allein auf den Markt als Steuerungsmechanismus verlassen, wie bei den Maßnahmen in DOM/MOD, sondern er muss stärker proaktiv bei der Gestaltung nachhaltigkeitsfördernder Rahmenbedingungen und Maßnahmen eingreifen, wie bei den Maßnahmen in MOD/ REGIO. Generelles Ziel muss es sein, wesentliche nicht-nachhaltige Aktivitäten - Energieverbrauch, Emissionen, Flächenverbrauch oder Substitution von Arbeit durch Kapital - finanziell stärker zu belasten. Mit den Einnahmen hieraus könnten die Behebung von Umweltproblemen, Kompensationen für negativ Betroffene, die Schaffung von Arbeitsplätzen, notwendige Investitionen im Bildungsbereich und die Unterstïtzung von Entwicklungsländern finanziert sowie die sozialen Sicherungssysteme armutsfest gemacht werden. Es wird deshalb ein tiefgreifender nachhaltigkeitsorientierter Umbau des bestehenden Steuer- und Abgabensystems für notwendig erachtet. Ein isoliertes Angehen einzelner Probleme steht, so die Studie, nicht nur im Widerspruch zum einem integrativen Nachhaltigkeitskonzept, sondern es würde auch die Erreichung der Ziele in ihrer Gesamtheit verhindern.

\section{Anmerkungen}

(1) Coenen, R./ Grunwald, A. (Hg.): Nachhaltigkeitsprobleme in Deutschland. Analyse und Lösungsstrategien, Berlin 2003.

(2) Kopfmüller, J. et al.: Nachhaltige Entwicklung integrativ betrachtet. Konstitutive Elemente, Regeln, Indikatoren, Berlin 2001.

\section{Die Autorlnnen}

Reinhard Coenen, Juliane Jörissen und Jürgen Kopfmüller sind wissenschaftliche Mitarbeiter des Instituts für Technikfolgenabschätzung und Systemanalyse des Forschungszentrums Karlsruhe in der Helmholtz-Gemeinschaft (ITAS).

Kontakt: ITAS, Hermann-von-Helmholtz-Platz I,

76344 Eggenstein-Leopoldshafen, Tel. 07247-

822501, E-Mail: kopfmueller@itas.fzk.de. 
(c) 20I0 Authors; licensee IÖW and oekom verlag. This is an article distributed under the terms of the Creative Commons Attribution Non-Commercial No Derivates License (http://creativecommons.org/licenses/by-nc-nd/3.o/), which permits unrestricted use, distribution, and reproduction in any medium, provided the original work is properly cited. 